\title{
Gastrografin-based conservative approach to small bowel obstructions: too far from a daily use
}

\author{
Mirko Barone*, Barbara Leone, Giuseppe Cipollone, Felice Mucilli \\ Department of General and Thoracic Surgery, University Hospital of Chieti, Chieti, Italy
}

\section{Dear Editor,}

As known, small bowel obstruction is one of the most common emergencies in general surgery carrying a not negligible rate of morbidity and financial expenditures as far as high social impact (1). Peritoneal adhesions are the leading cause of intestinal obstruction up to $74 \%$ of cases. In spite of increasing incidence worldwide, current management still presents some "blind corners", whose aspects still claim debate. In fact, notwithstanding guidelines, some issues such as limits of conservative approaches, efficacy and safety of nonoperative strategies as well as the risk of a delayed surgery arise both medical and somewhat legal concerns with an increased mortality rate from $3 \%$ to $5 \%$ for simple obstructions and $30 \%$ for complicated ones (2). Diagnostic and therapeutic aspects seem to be embraced by the use of standardized Gastrografin-based protocols, whose findings are still conflicting (3). Gastrografin, a hyperosmolar oral watersoluble and ionic mixture of sodium diatrizoate and meglumine diatrizoate, promotes shifting of intraluminal fluids and facilitates bowel motility. Its use carries both diagnostic and therapeutic issues. In fact, as CT contrast agent, it facilitates obstruction identification (spatial resolution) and severity (dynamic resolution). Moreover, according to its biochemical properties, gastrografin ideally promotes mechanical ileus resolution. However, evidences are still unclear resulting in conflicting reports. Biondo et al (4) noticed that contrast agents reduce the operative rate by $35 \%$ with a subsequent increased success of conservative approaches of about 7\%. Similarly, Di Saverio et al (5) reported a reduction of surgery rates ( $18.5 \%$ vs $45 \%)$, hospital stay (4.6 vs 7.8 days) and time- to-resolution of adhesive syndromes (6.9 vs 43 hours). Rahmani et al (6), in a RCT enrolling 84 adhesive patients, reported only a statistically significant reduction in hospital stay ( 2.69 vs $4.67, P=0.000)$ associated with a reduced, but without any level of evidence, need for surgery $(p=0.07)$. Similar results were published by Srinivasa et al (7) who, in a 10 -year retrospective analysis, reported no difference in operative rate between groups $(5.2 \%$ vs $9.2 \%, P=0.10)$. In spite of these results, Abbas et al (8) showed no reduction in surgery rates, though a reduction in hospital stay was noticed. And so, is it really effective? Are there any biases influencing results or indications? According to these evidences, also the recent World Society of Emergency Surgery Guidelines (9) describes some therapeutic approaches though contrast agents applications still reply only for diagnostic purposes and no levels of evidences have been reported in this regard.

Another aspect to consider is the proper indication for a Gastrografin protocol, which could be reserved only in patients without signs of strangulations or closed loop obstructions. For these reasons, related efficacy and safety must be restricted to selected cohorts of patients (i.e. patients without thyropathies due to the risk of exacerbation), as being far from a universal adoption. Moreover, though rare, fearful and life-threatening side effects have also been described with the administration of oral contrast agents, such as haemorrhagic gastritis, bleedings, bowel necrosis or perforations (10). For these reasons, daily use prior to a careful clinical and radiological evaluation should be discouraged, especially in frail elderly patients in which an induced electrolyte imbalance could exacerbate a pre-existent fragile condition, such as in case

Received: 22 October 2018; Accepted: 19 January 2019; Published online: 28 January 2019

*Corresponding author: Mirko Barone, MD; Department of General and Thoracic Surgery, University "G. d'Annunzio" of Chieti (Italy). Tel: +39 0871 358289 , Fax: +390871 358220, E-mail: mir87mb@libero.it

Competing interests: None

Funding information: None.

Citation: Barone M, Leone B, Cipollone G, Mucilli F. Gastrografin-based conservative approach to small bowel obstructions: too far from a daily use. Journal of Emergency Practice and Trauma 2020; 6(1): 1-2. doi: 10.15171/jept.2019.01. 
of renal failure or chronic hepatopathies. Protocols and inclusion criteria should be set according to comorbidities. However, aren't most of small bowel obstruction cases in elderly patients? We should not overcome current and proper indications as sometimes off-label approaches could reserve unpleasant surprises.

\section{Ethical issues}

Not applicable.

\section{Authors' contributions}

Authors contributed equally to this work.

\section{References}

1. Cooper JM, Thirlby RC. Small Bowel Obstruction. Curr Treat Options Gastroenterol 2002; 5(1): 3-8.

2. Ellis $\mathrm{H}$. The clinical significance of adhesions: focus on intestinal obstruction. Eur J Surg Suppl 1997; (577): 5-9.

3. Choi HK, Chu KW, Law WL. Therapeutic value of gastrografin in adhesive small bowel obstruction after unsuccessful conservative treatment: a prospective randomized trial. Ann Surg 2002; 236(1): 1-6.

4. Biondo S, Pares D, Mora L, Marti Rague J, Kreisler E, Jaurrieta E. Randomized clinical study of Gastrografin administration in patients with adhesive small bowel obstruction. Br J Surg 2003; 90(5): 542-6. doi: 10.1002/ bjs. 4150 .

5. Di Saverio S, Catena F, Ansaloni L, Gavioli M, Valentino M,
Pinna AD. Water-soluble contrast medium (gastrografin) value in adhesive small intestine obstruction (ASIO): a prospective, randomized, controlled, clinical trial. World J Surg 2008; 32(10): 2293-304. doi: 10.1007/s00268-0089694-6.

6. Rahmani N, Mohammadpour RA, Khoshnood P, Ahmadi A, Assadpour S. Prospective evaluation of oral gastrografin $\left({ }^{\circledR}\right)$ in the management of postoperative adhesive small bowel obstruction. Indian J Surg 2013; 75(3): 195-9. doi: 10.1007/ s12262-012-0479-7.

7. Srinivasa S, Thakore N, Abbas S, Mahmood M, Kahokehr AA, Hill AG. Impact of gastrografin in clinical practice in the management of adhesive small bowel obstruction. Can J Surg 2011; 54(2): 123-7. doi: 10.1503/cjs.024009.

8. Abbas S, Bissett IP, Parry BR. Oral water soluble contrast for the management of adhesive small bowel obstruction. Cochrane Database Syst Rev 2007; (3): Cd004651. doi: 10.1002/14651858.CD004651.pub3.

9. Ten Broek RPG, Krielen P, Di Saverio S, Coccolini F, Biffl WL, Ansaloni L, et al. Bologna guidelines for diagnosis and management of adhesive small bowel obstruction (ASBO): 2017 update of the evidence-based guidelines from the world society of emergency surgery ASBO working group. World J Emerg Surg 2018; 13: 24. doi: 10.1186/s13017-0180185-2.

10. Guy S, Al Askari M. Haemorrhagic gastritis following Gastrografin administration for adhesive small bowel obstruction: a case report of a rare outcome. Int J Surg Case Rep 2017; 33: 51-4. doi: 10.1016/j.ijscr.2017.02.030. 